\title{
Neonatal DTI early after birth predicts motor outcome in preterm infants with periventricular hemorrhagic infarction
}

Elise Roze', Manon J. Benders' ${ }^{1}$, Karina J. Kersbergen'1, Niek E. van der Aa', Floris Groenendaal', Ingrid C. van Haastert', Alexander Leemans ${ }^{2}$ and Linda S. de Vries ${ }^{1}$

BACKGROUND: To determine the association between early neonatal diffusion tensor imaging (DTI) and the development of unilateral spastic cerebral palsy (USCP) in preterm infants with periventricular hemorrhagic infarction (PVHI).

METHODS: Preterm infants with PVHI were assessed with early ( $\leq 4 \mathrm{wk}$ after birth) and term-equivalent age MRI-DTI. Involvement of corticospinal tracts was assessed by visual assessment of the posterior limb of the internal capsule (PLIC) on DTI (classified asymmetrical, equivocal, or symmetrical) and by an atlas-based approach calculating fractional anisotropy asymmetry index in the PLIC. Motor outcome was assessed at $\geq 15$ mo corrected age.

RESULTS: Seven out of 23 infants with PVHI developed USCP. Their PLIC was visually scored as asymmetrical in 6 and equivocal in 1 on the early DTI. Thirteen out of 16 infants with a symmetrical motor development had a symmetrical PLIC on early DTI, the remaining 3 were equivocal. All infants with USCP had a fractional anisotropy asymmetry index of $>0.05$ (optimal cut-off value) on early DTI. In infants with a symmetrical motor development $(n=16), 14$ had an asymmetry index $\leq 0.05$ while 2 had an index $>0.05$.

CONCLUSION: DTI in preterm infants with PVHI within a few weeks after birth is associated with later motor development.

$\mathbf{P}$ eriventricular hemorrhagic infarction (PVHI) is a serious type of brain injury that occurs in around $1-3 \%$ of preterm infants with a gestational age below $37 \mathrm{wk}(1-3)$. It is a complication of a germinal matrix hemorrhage that develops due to impaired venous drainage of the medullary veins in the periventricular white matter and may have an adverse effect on corticospinal tract (CST) development (4). PVHI leads in about $50-85 \%$ of infants to, mainly unilateral, spastic cerebral palsy (USCP) $(2,3,5)$. The diagnosis is made with cranial ultrasound (cUS) and prediction of cerebral palsy is possible in most but not all infants taken the site and size of the lesion into account (5).

MRI studies performed at term-equivalent age (TEA) will reliably predict motor outcome with asymmetry in myelination of the posterior limb of the internal capsule (PLIC) due to Wallerian degeneration being almost invariably associated with the development of USCP (6).

Diffusion tensor imaging (DTI) allows the study of microstructural properties of the white matter (7). To the best of our knowledge, it has not yet been studied whether early disturbances in CST integrity shortly after the onset of PVHI relate to later development of USCP. In addition, the prognostic value of DTI for early prediction of USCP in infants with PVHI is still unknown.

The aim of this study was to determine the association between MRI-DTI within $4 \mathrm{wk}$ after birth and the development of USCP in preterm infants with PVHI.

\section{RESULTS}

\section{Patients}

The clinical characteristics and motor outcome of the study group $(n=23)$ are summarized in Table 1 . The infants who developed cerebral palsy $(n=7,30 \%)$ all had USCP.

\section{Cranial Ultrasound Findings}

In 16 infants with a normal motor development, the cUS on which the PVHI was first diagnosed was scored as normal in 13 infants, predictive of USCP in 1 and equivocal in 2 infants. The cUS at TEA was scored normal in 13 infants and predictive of USCP in 1 infant. Of two infants, no cUS was available at TEA.

In seven infants with USCP, the cUS was predictive of USCP in all infants, both on the early cUS as on the cUS at TEA. The predictive value of cUS for development of USCP is shown in Table 2.

\section{Early DTI Findings in Relation to Outcome}

The time between the cUS on which the PVHI was first seen and the early DTI scan was median $12 \mathrm{~d}$ (range $0-22 \mathrm{~d}$ ). At the early DTI scan, the median postmenstrual age of the 23 infants was $31.3 \mathrm{wk}$ (range 29.3-36.4 wk).

\section{Visual Assessment of Direction Encoded FA Maps}

Visual qualitative assessment of the PLIC was performed on direction encoded fractional anisotropy (FA) maps obtained in 
the early neonatal period (Figure 1). Cohen's $\kappa$ coefficient for inter-observer variability for DTI assessment was $k=0.93$. Six of the seven infants with USCP were scored as asymmetrical

Table 1. Patient characteristics

\begin{tabular}{lc}
\hline & Infants with PVHI \\
\hline Number & $n=23$ \\
Males/females & $9 / 14$ \\
Gestational age (wk) & $28.9(25.7-34.6)$ \\
Birth weight (g) & $1,200(650-1,950)$ \\
Ultrasound characteristics & \\
Grade I GMH & $n=2(9)$ \\
Grade II GMH & $n=5(22)$ \\
Grade III GMH & $n=15(65)$ \\
Bilateral GMH & $n=12(52)$ \\
PHVD & $n=9(39)$ \\
Motor outcome & \\
Age at follow-up (months) & \\
Cerebral palsy & $29(15-67)$ \\
\hline
\end{tabular}

Data are given as number (percentage) or median (minimum-maximum).

$\mathrm{GMH}$, germinal matrix hemorrhage; PHVD, posthemorrhagic ventricular dilatation;

$\mathrm{PVH}$, periventricular hemorrhagic infarction.

aCorrected for prematurity. and 1 as equivocal. In 13 of the 16 children with a normal motor development, the PLIC was scored symmetrical whilst the remaining 3 were scored as equivocal. The predictive value is shown in Table 2.

\section{FA Analysis}

Based on the receiver operating characteristics curve (Figure 2) the Youden index for most optimal prediction of cerebral palsy was an asymmetry index of $>0.05$ (area under the curve 0.93, 95\% CI 0.82-1.00). All of the infants with USCP had an asymmetry index of $>0.05$ (Figure 3a). In infants with a symmetrical development $(n=16), 14$ had an asymmetry index $\leq 0.05$ while 2 had an asymmetry index of $>0.05$ (Figure 3a). One infant with a symmetrical motor development had an asymmetry index of -0.18 , which indicates higher FA on the ipsilateral hemisphere.

Of the four infants that were classified with an equivocal PLIC by visual assessment on FA maps, the asymmetry index in FA was 0.11 in the infant that developed USCP and 0.00 , -0.01 and -0.18 in the three infants who had a symmetrical development.

Of the two infants with an asymmetry index of $>0.05$ with a subsequent symmetrical development, visual assessment was symmetrical in both. In these two infants, MRI-DTI scans at TEA were available. One infant normalized at TEA (asymmetry index $<0.05$ with symmetry on visual assessment),

Table 2. Predictive value of different imaging techniques

\begin{tabular}{|c|c|c|c|c|}
\hline & Sensitivity & Specificity & $\begin{array}{c}\text { Positive } \\
\text { predictive value }\end{array}$ & $\begin{array}{c}\text { Negative } \\
\text { predictive value }\end{array}$ \\
\hline cUS early after birth & $100 \%(56-100 \%)$ & $94 \%(68-100 \%)$ & $88 \%(47-99 \%)$ & $100 \%(75-100 \%)$ \\
\hline Earlya visual DTI assessment & $86 \%(42-99 \%)$ & $100 \%(76-100 \%)$ & $100 \%(52-100 \%)$ & $94 \%(69-100 \%)$ \\
\hline Early asymmetry index FA & $100 \%(56-100 \%)$ & $88 \%(60-98 \%)$ & $78 \%(40-96 \%)$ & $100 \%(73-100 \%)$ \\
\hline
\end{tabular}

Data are given as percentage $(95 \% \mathrm{CI})$. Normal and equivocal scans were taken together in the calculations.

DTI, diffusion tensor imaging; FA, fractional anisotropy; MRI, magnetic resonance imaging; TEA, term-equivalent age.

aEarly refers to a DTI scan within 4 wk after birth.
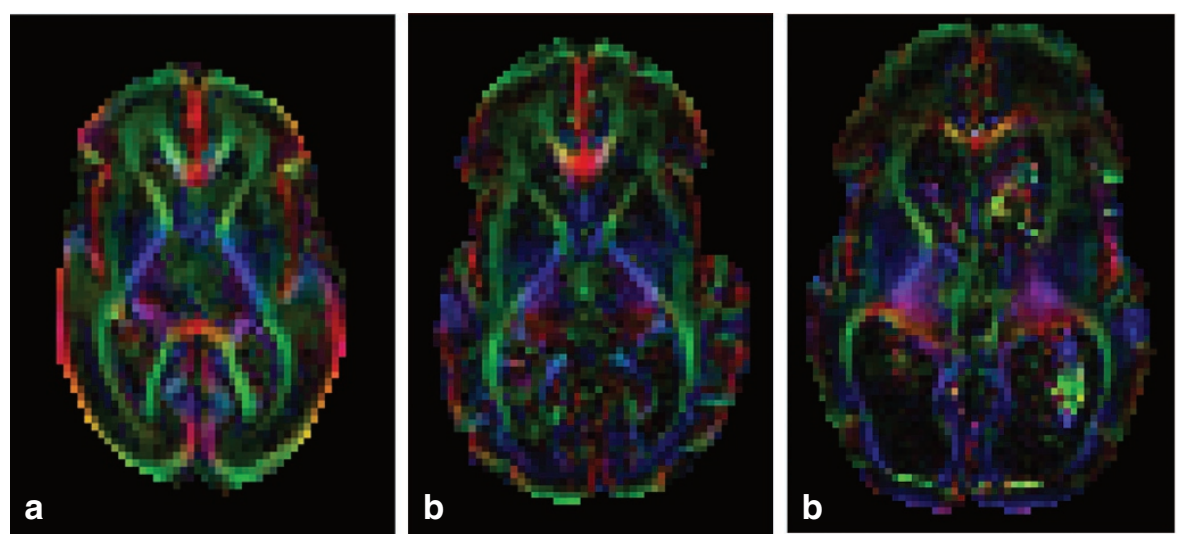

Figure 1. Visual assessment of the PLIC on fractional anisotropy maps. Symmetrical (a), equivocal (b), and asymmetrical (c) posterior limb of the internal capsule on direction encoded fractional anisotropy maps from the early neonatal period in infants with periventricular hemorrhagic infarction. 


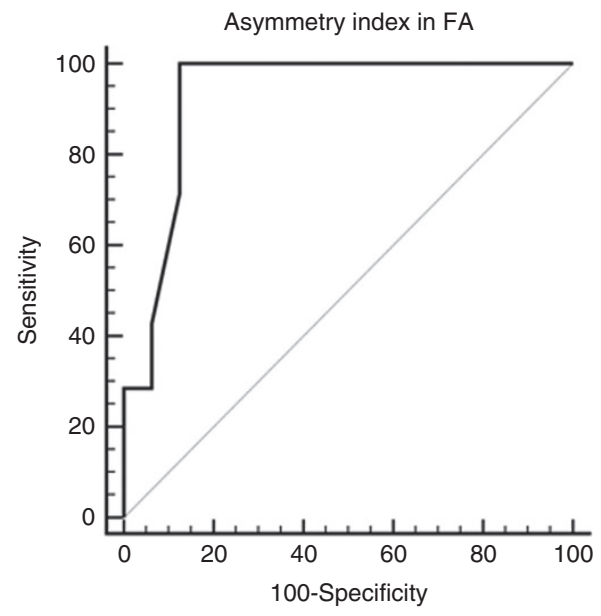

Figure 2. Receiver operating characteristics curve for the asymmetry index in fractional anisotropy of the posterior limb of the internal capsule and development of cerebral palsy.

while the other remained asymmetrical at TEA (asymmetry index $>0.05$ and equivocal on visual assessment).

Regarding the other diffusion parameters of the PLIC, radial diffusivity (RD) was also found to be related to cerebral palsy. The Youden index for RD was an asymmetry of $\leq-0.04$ (area under the curve $0.88,95 \% \mathrm{CI} 0.72-1.00$ ), the asymmetry index for $\mathrm{RD}$ is shown in Figure $3 \mathrm{~b}$. Mean diffusivity and axial diffusivity were not related to outcome.

\section{Early Neonatal DTI Compared to Conventional MRI at TEA}

Visual assessment of myelination of the PLIC on T1-weighted sequence was performed at TEA. All six infants with an asymmetry of the PLIC on visual assessment of the direction encoded FA maps in the early neonatal period were found to have asymmetrical myelination of the PLIC at TEA. In those with an early equivocal PLIC on DTI $(n=4)$, myelination of the PLIC was asymmetrical in 1 who subsequently developed USCP and symmetrical in the remaining three who showed a normal early motor development. Eleven of the 13 infants with a symmetrical PLIC in the early neonatal period were found to have a symmetrical PLIC on T1 at TEA while 2 were judged equivocal. All these infants had a symmetrical development.

The two infants with an abnormal asymmetry index of FA $(>0.05)$ in the early period and a normal motor outcome, had a symmetrical PLIC at TEA on T1 weighted imaging.

In Figure 4, the results on the subsequent neuro-imaging tests are graphically shown in order to see if certain imaging techniques may not be needed to correctly predict outcome. All infants with symmetrical or equivocal cUS findings had a symmetrical development (Figure $4 \mathrm{~b}$ ) and would not have required additional MRI. Seven of the eight infants with an asymmetrical cUS had an asymmetrical development and TEA-MRI was required to correctly predict outcome.

All 13 infants with a visual symmetry on the DTI had a symmetrical development and the 6 infants with visual asymmetry had an asymmetrical development, thus no further assessment was needed (Figure 4a). In the remaining four infants with an equivocal visual DTI, the T1 at term equivalent age showed

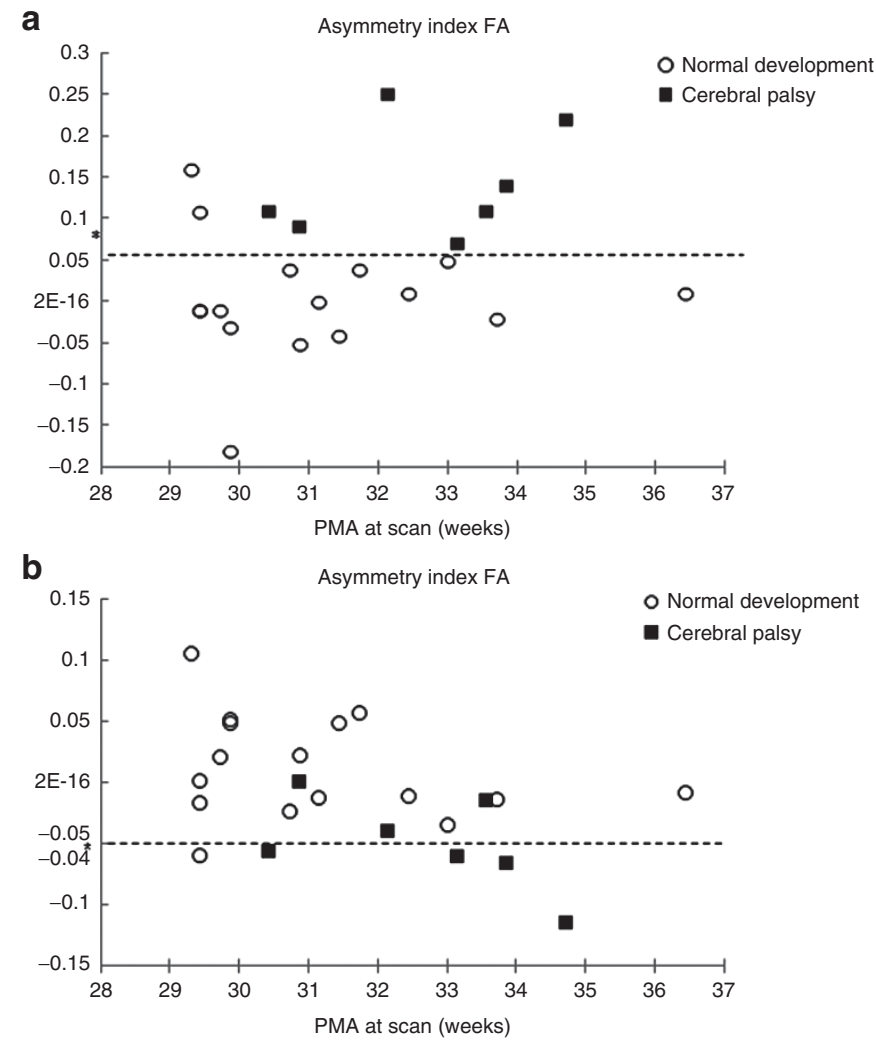

Figure 3. The asymmetry index of diffusion parameters in the PLIC in relation to outcome. Asymmetry index in the posterior limb of the internal capsule in fractional anisotropy (a) and radial diffusivity (b) in infants with periventricular hemorrhagic infarction with normal outcome and cerebral palsy. *Indicates optimal cut-off for prediction of cerebral palsy.

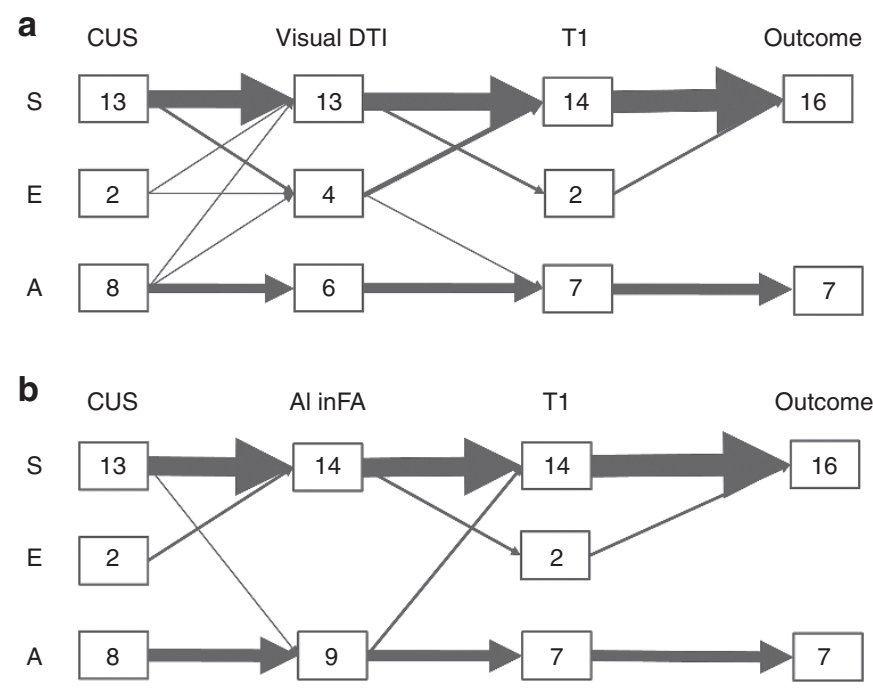

Figure 4. Sequential imaging techniques in relation to outcome. Results on subsequent imaging techniques including visual DTI judgment (a) and asymmetry index in FA (b). a, asymmetrical; Al, asymmetry index; cUS, cranial ultrasound scan; DTI, diffusion tensor imaging; e, equivocal; FA, fractional anisotropy; s, symmetrical.

symmetrical myelination of the PLIC with a symmetrical outcome in three infants and an asymmetry in myelination in the other infant with subsequent development of USCP. 
With regard to the asymmetry index in FA on DTI (Figure $4 \mathrm{~b}$ ), all 14 infants with a normal asymmetry index showed a symmetrical development, even though myelination of the PLIC was considered equivocal in two infants at TEA. Two of the nine infants with an abnormal asymmetry index had symmetrical myelination of the PLIC with a subsequent symmetrical development.

\section{Cognitive Outcome}

Cognitive outcome was measured in 22 out of 23 infants. Of them, 18 infants (82\%) scored within 1 SD of the norm (cognitive quotient 85-110), while 4 infants (18\%) scored in the subclinical range (cognitive quotient $70-85$ ). None of the infants had a score in the clinical range (cognitive quotient $<70$ ). Four of the seven infants with cerebral palsy scored in the subclinical range, and their cognitive quotient was significantly worse than in infants with normal development (median cognitive quotient 83 vs. $95, P<0.05)$.

\section{DISCUSSION}

This study shows that early neonatal DTI obtained within $4 \mathrm{wk}$ after birth is predictive of motor outcome in preterm infants with PVHI. All but one of the infants with USCP showed an asymmetry on the early direction encoded FA maps. One infant with USCP was equivocal on visual inspection on the early DTI, but had asymmetrical myelination at TEA. A further three infants who also were equivocal on early direction encoded FA maps but without subsequent motor problems had symmetrical myelination of the PLIC at TEA. Using an atlas-based approach, it was shown that an asymmetry index in FA $>0.05$ was noted in all infants with USCP. In another two infants with a FA $>0.05$ but a normal motor outcome, myelination of the PLIC was symmetrical at TEA, suggesting that early DTI helps to predict motor outcome early, but assessment of myelination at TEA may still be required. Asymmetry in RD was also related to later USCP although with a lower sensitivity than FA.

Several previous studies have tried to identify early imaging characteristics in PVHI with prognostic value for outcome, with cUS being the most extensively studied imaging modality. Although cUS has been shown to be a highly reliable technique to detect PVHI $(8,9)$, its sensitivity to detect CST involvement and its prognostic value for USCP has not been uniformly established. One study found that frontal involvement was particularly associated with abnormal neuromotor development (10), whereas other studies have shown that a large PVHI involving more than one brain region or located posterior to the trigone is associated with development of USCP (5). In the present study, a high positive and negative predictive value was found when the PVHI was classified into either anterior or posterior to the trigone.

Asymmetry in signal intensity within the PLIC on the TEA MRI has been shown to be a reliable early predictor of USCP in infants with PVHI (6), as is confirmed in the present study. This important finding of asymmetry in myelination will however not become apparent until TEA, since myelination is not evident in the PLIC on MR imaging before $36 \mathrm{wk}$ gestation (11). This is in line with histopathological studies that report the onset of myelination in the PLIC to occur between 32 and 36 wk gestation.

The mechanism of axonal injury following PVHI, Wallerian degeneration, begins within a week after the injury and progresses through several pathophysiologically distinct stages over the next 3-6 mo (12). So far, it has remained unclear whether early pathophysiological changes in CST integrity in the neonatal period after emergence of PVHI may be an early sign of axonal injury and be associated with disturbances in myelin sheath development.

Changes in water diffusion following this process of Wallerian degeneration can be visualized with DTI (13). Previous studies have shown that asymmetries in FA of the CST after PVHI and arterial stroke at TEA indicate CST injury that is associated with the development of USCP (14-16). The present study adds that in preterm infants with a PVHI, asymmetry in FA can be detected within weeks after the onset of PVHI reflecting early alterations in CST integrity that relate to later motor outcome. This asymmetry in FA may be due to cytotoxic edema and increased intracellular water from focal axonal swelling that occurs in the early stage of Wallerian degeneration (17).

In the present study, two different techniques for DTI assessment on early MRI within $4 \mathrm{wk}$ after birth were used, i.e., visual assessment and an atlas-based approach. To the best of our knowledge, this study is the first to describe visually detected asymmetries in FA on early DTI after PVHI. Previous studies on diffusion-weighted imaging in term born infants with arterial stroke showed that visually detected abnormalities in signal intensity at the level of the PLIC and cerebral peduncle are suggestive of pre-Wallerian degeneration. They were noted to be predictive of subsequent Wallerian degeneration and an adverse motor outcome $(14,18,19)$. The present study adds that changes in FA after PVHI can already be visually detected at a very early age.

Following an atlas-based approach, an asymmetry index cut-off of $>0.05$ was found to be predictive of development of USCP. Previous studies reporting on asymmetry indices at TEA found slightly higher values related to USCP at TEA and $11 \mathrm{y}$ of age $(16,20)$. Presumably the asymmetry will become more pronounced at a later age when myelination progresses (20). In addition, an increased $\mathrm{RD}$ on the affected side was found predictive of later development of USCP. This is in line with previous studies at TEA also showing asymmetry in RD in infants with PVHI that developed cerebral palsy $(16,21)$. In the present study, however, $\mathrm{RD}$ was not of additional value to asymmetry in FA.

In our cohort, $30 \%$ of the infants with PVHI developed USCP, which is lower than reported previously (47-90\%) $(2,3,10,22)$. This might be explained by differences in baseline characteristics of the infants, especially the size and site of the PVHI, and differences in redirection of care.

The strength of the present study was the relatively large number of infants with PVHI that were scanned in both the 
early neonatal period and at TEA. This enabled us to study the course of CST injury due to PVHI.

This study is subject to potential limitations such as the use of different MRI-DTI protocols. This may be of influence on a comparison of absolute FA values between infants. We tried to overcome this problem by using an asymmetry index to compare ipsi- and contralateral CSTs within infants. In addition, the presence of large brain lesions may influence the accuracy of the registration in an atlas based approach. Although a quality check of the registration was done for each infant, we cannot completely rule out this effect. We determined a cutoff for asymmetry in FA in the same population as in which this cutoff was tested. In accordance with the TRIPOD statement, further research is needed to independently validate this cutoff in a control population of preterm infants without brain lesions (23).

Another limitation was the relatively young age at follow-up. It is known that a definite diagnosis of USCP cannot yet be reliably made at the age of $18 \mathrm{mo}$ and a mild USCP might still develop in some of these infants (24). Although a majority of the current cohort was $\geq 2 y$ of age, time must tell whether the motor assessment of the youngest infants will remain stable over time.

The infants were still too young to perform functional neuroimaging. It is of interest to assess whether the infants with an early asymmetry index of $>0.05$ but with normal $(n=2)$ early motor outcome, will still develop an asymmetry or will later have an altered pattern of cortical reorganization of motor function (25).

In conclusion, in preterm infants with PVHI, a reliable prediction of motor outcome can be made in most infants by neonatal DTI within $4 \mathrm{wk}$ after birth. Early identification of infants at risk for developing cerebral palsy may be of help in selecting candidates for potential neuroprotective intervention strategies that aim to improve outcome.

\section{METHODS}

\section{Patients}

All preterm infants admitted to the Neonatal Intensive Care Unit of the Wilhelmina Children's Hospital between 2007-2012, with a gestational age $\leq 34 \mathrm{wk}$, and a PVHI diagnosed using cUS were included. In addition, the following inclusion criteria were used: MRI-DTI performed in the early neonatal period (i.e., within $4 \mathrm{wk}$ after birth), and again at TEA. Patients with chromosomal and congenital anomalies, and bilateral PVHI were excluded (Figure 5).

No permission was required from the hospital's medical ethics committee for this retrospective, anonymous data analysis.

\section{Imaging Protocol}

cUS, using a broadband transducer (5-8.5 MHz, Aplio XG scanner (Toshiba medical system, Zoetermeer, The Netherlands)) was performed as standard of care. The first cUS was performed as soon as possible after admission, repeated two to three times during the first week and once a week until discharge, and again at TEA. Prediction of motor development was done based on the first week cUS when the PVHI was diagnosed and on the cUS at TEA, when available. If the PVHI was localized anterior to the trigone the cUS was scored as predictive of normal development. If it was localized posterior to the trigone in the parietal periventricular white matter, it was scored as predictive of USCP (5). When it was unclear whether the PVHI was located anterior or posterior to the trigone, it was scored as equivocal.

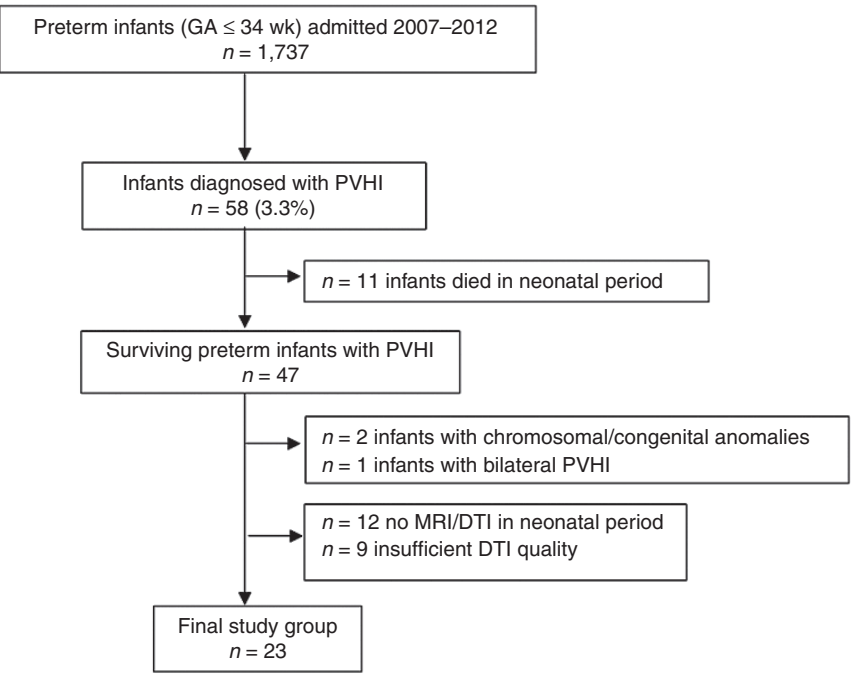

Figure 5. Flow chart of composition of final study group of preterm infants with periventricular hemorrhagic infarction.

Visual inspection of the site of the PVHI in relation to the trigone was performed by a neonatologist with longstanding experience in cUS $(\mathrm{LdV})$. All patients underwent serial cUS prior to the MRI.

MRI scans were acquired on either a $1.5 \mathrm{~T}$ or 3T Philips MR system (Philips, Best, The Netherlands) and included T1- and T2-weighted imaging. Single-shot echo planar DTI was acquired in either 32 or 45 noncollinear directions with one of the following scanning protocols;1.5T, 32 directions: repetition time (TR): $6.817 \mathrm{~ms}$, echo time $(\mathrm{TE})=87 \mathrm{~ms}$, voxel size $=1.98 \times 1.98 \times 2 \mathrm{~mm}$, b value $800 \mathrm{~s} / \mathrm{mm}^{2}$, SENSE factor of 2.5 ; 3T, 32 directions: TR: $5.685 \mathrm{~ms}$, TE $=70 \mathrm{~ms}$, voxel size $=1.41 \times 1.41 \times 2 \mathrm{~mm}$, b value $800 \mathrm{~s} / \mathrm{mm}^{2}$, SENSE factor of $2 ; 3 \mathrm{~T}$, 45 directions: TR: $6.500 \mathrm{~ms}, \mathrm{TE}=80 \mathrm{~ms}$, voxel size $=2 \times 2 \times 2 \mathrm{~mm}$, b value $800 \mathrm{~s} / \mathrm{mm}^{2}$, SENSE factor of 1.4. DTI scans of insufficient quality due to motion artifacts or technical errors in reconstruction were excluded.

\section{Imaging Assessment}

Diffusion properties of the CST were assessed with the ExploreDTI diffusion MRI toolbox (www.exploredti.com) (26). Postprocessing included the following steps: (i) correction for subject motion and eddy current induced distortions (27); (ii) tensor estimation using the REKINDLE approach for outlier detection (28) with iteratively reweighted linear least squares estimation after identification and removal of data outliers (29); and (iii) automated atlas-based analysis with the neonatal atlas from Oishi et al. (30) (template and parcellated atlas regions are availableathttp://cmrm.med.jhmi.edu, center of Magnetic Resonance Microimaging, Baltimore) using affine and elastic registration based on "elastix" (31). After postprocessing, the DTI data were visually checked in terms of quality of tensor estimation and quality of registration to the atlas. After these steps, FA, axial diffusivity, $\mathrm{RD}$, and mean diffusivity values were calculated at the level of the PLIC, as provided by the Oishi atlas (30).

In addition, we used a qualitative method based on visual inspection of the PLIC on direction encoded FA maps to assess asymmetry. The integrity of the PLIC was assessed at the slice where the angle between the anterior limb of the internal capsule and the PLIC could be most clearly visualized. With visual inspection on direction encoded FA maps, the PLIC was classified as symmetrical, equivocal, or asymmetrical according to number of voxels (length) and intensity (brightness). This was also done for the conventional T1-weighted MRI at TEA. Visual inspection of DTI and T1weighted MRI was performed by two neonatologists with longstanding experience in MRI (L.S.dV., M.J.B.). In case the experts disagreed with one another, a third expert (F.G.) was consulted and the scans in question were reevaluated together. In all cases, a consensus was reached on the final score. The assessors were blinded to the infant's outcome. 


\section{Follow-Up}

The infants were seen in the follow-up clinic, for assessment of motor and cognitive outcome up to $5 \mathrm{y}$ of age. Motor outcome was assessed with an age-specific neurological examination. The presence or absence of USCP was determined at 15 mo corrected age or beyond, with median age 29 mo (range 15-67), following the criteria of Bax et al. (32). Cognitive outcome was assessed with either the Griffiths Mental Development Scales, the cognitive scale of the Bayley Scales of Infant Development (BSID-III), or the Wechsler Preschool and Primary Scale of Intelligence (WPPSI-III). It was assessed at a median age of $27 \mathrm{mo}$ (range 24-68 mo).

\section{Statistical Analyses}

Cohen's $\kappa$ for interobserver variability was calculated for the visual DTI assessment, determining agreement in an asymmetrical vs. a normal or equivocal PLIC. An asymmetry index in FA was calculated using (mean FA contralateral PLIC - mean FA ipsilateral PLIC)/ (mean FA contralateral PLIC + mean FA ipsilateral PLIC) (33), in which ipsilateral refers to the PLIC of the hemisphere where the PVHI was localized. A receiver operating characteristics curve was created to assess the predictive ability of the asymmetry index for development of cerebral palsy. The "optimal" cut-off value of the asymmetry index for prediction of cerebral palsy was determined by the maximal Youden index (34), with MedCalc 14.8.1 software (MedCalc Software, Ostend, Belgium).

In addition, the positive predictive value, negative predictive value, sensitivity, and specificity of the different imaging techniques were calculated by comparing infants with an asymmetrical PLIC to those with a symmetrical or equivocal PLIC respectively.

Disclosures: All authors declare that they have nothing to disclose, financially or otherwise. There is no conflict of interest. This study is not the result of a clinical trial.

\section{REFERENCES}

1. Hamrick SE, Miller SP, Leonard C, et al. Trends in severe brain injury and neurodevelopmental outcome in premature newborn infants: the role of cystic periventricular leukomalacia. J Pediatr 2004;145:593-9.

2. Roze E, Kerstjens JM, Maathuis CG, ter Horst HJ, Bos AF. Risk factors for adverse outcome in preterm infants with periventricular hemorrhagic infarction. Pediatrics 2008;122:e46-52.

3. Maitre NL, Marshall DD, Price WA, et al. Neurodevelopmental outcome of infants with unilateral or bilateral periventricular hemorrhagic infarction. Pediatrics 2009;124:e1153-60.

4. Volpe JJ. Brain injury in premature infants: a complex amalgam of destructive and developmental disturbances. Lancet Neurol 2009;8:110-24.

5. Rademaker KJ, Groenendaal F, Jansen GH, Eken P, de Vries LS. Unilateral haemorrhagic parenchymal lesions in the preterm infant: shape, site and prognosis. Acta Paediatr 1994;83:602-8.

6. De Vries LS, Groenendaal F, van Haastert IC, Eken P, Rademaker KJ, Meiners LC. Asymmetrical myelination of the posterior limb of the internal capsule in infants with periventricular haemorrhagic infarction: an early predictor of hemiplegia. Neuropediatrics 1999;30:314-9.

7. Hüppi PS, Dubois J. Diffusion tensor imaging of brain development. Semin Fetal Neonatal Med 2006;11:489-97.

8. Bass WT, Jones MA, White LE, Montgomery TR, Aiello F 3rd, Karlowicz MG. Ultrasonographic differential diagnosis and neurodevelopmental outcome of cerebral white matter lesions in premature infants. J Perinatol 1999;19:330-6.

9. de Vries LS, Roelants-van Rijn AM, Rademaker KJ, Van Haastert IC, Beek FJ, Groenendaal F. Unilateral parenchymal haemorrhagic infarction in the preterm infant. Eur J Paediatr Neurol 2001;5:139-49.

10. Bassan $\mathrm{H}$, Benson CB, Limperopoulos C, et al. Ultrasonographic features and severity scoring of periventricular hemorrhagic infarction in relation to risk factors and outcome. Pediatrics 2006;117:2111-8.

11. Sie LT, van der Knaap MS, van Wezel-Meijler G, Valk J. MRI assessment of myelination of motor and sensory pathways in the brain of preterm and term-born infants. Neuropediatrics 1997;28:97-105.
12. Aldskogius H, Kozlova EN. Central neuron-glial and glial-glial interactions following axon injury. Prog Neurobiol 1998;55:1-26.

13. Thomalla G, Glauche V, Koch MA, Beaulieu C, Weiller C, Röther J. Diffusion tensor imaging detects early Wallerian degeneration of the pyramidal tract after ischemic stroke. Neuroimage 2004;22:1767-74.

14. Domi T, deVeber G, Shroff M, Kouzmitcheva E, MacGregor DL, Kirton A. Corticospinal tract pre-wallerian degeneration: a novel outcome predictor for pediatric stroke on acute MRI. Stroke 2009;40:780-7.

15. van der Aa NE, Leemans A, Northington FJ, et al. Does diffusion tensor imaging-based tractography at 3 months of age contribute to the prediction of motor outcome after perinatal arterial ischemic stroke? Stroke 2011;42:3410-4.

16. Roze E, Harris PA, Ball G, et al. Tractography of the corticospinal tracts in infants with focal perinatal injury: comparison with normal controls and to motor development. Neuroradiology 2012;54:507-16.

17. Venkatasubramanian C, Kleinman JT, Fischbein NJ, et al. Natural history and prognostic value of corticospinal tract Wallerian degeneration in intracerebral hemorrhage. J Am Heart Assoc 2013;2:e000090.

18. Groenendaal F, Benders MJ, de Vries LS. Pre-wallerian degeneration in the neonatal brain following perinatal cerebral hypoxia-ischemia demonstrated with MRI. Semin Perinatol 2006;30:146-50.

19. Mercuri E, Cowan F, Rutherford M, Acolet D, Pennock J, Dubowitz L. Ischaemic and haemorrhagic brain lesions in newborns with seizures and normal Apgar scores. Arch Dis Child Fetal Neonatal Ed 1995;73: F67-74.

20. Tsao H, Pannek K, Fiori S, Boyd RN, Rose S. Reduced integrity of sensorimotor projections traversing the posterior limb of the internal capsule in children with congenital hemiparesis. Res Dev Disabil 2014;35:250-60.

21. Arichi T, Counsell SJ, Allievi AG, et al. The effects of hemorrhagic parenchymal infarction on the establishment of sensori-motor structural and functional connectivity in early infancy. Neuroradiology 2014;56: 985-94.

22. De Vries LS, Van Haastert IL, Rademaker KJ, Koopman C, Groenendaal F. Ultrasound abnormalities preceding cerebral palsy in high-risk preterm infants. J Pediatr 2004;144:815-20.

23. Collins GS, Reitsma JB, Altman DG, Moons KG. Transparent reporting of a multivariable prediction model for individual prognosis or diagnosis (TRIPOD): the TRIPOD Statement. BMC Med 2015;13:1.

24. Kuban KC, Leviton A. Cerebral palsy. N Engl J Med 1994;330:188-95.

25. van der Aa NE, Verhage $\mathrm{CH}$, Groenendaal F, et al. Neonatal neuroimaging predicts recruitment of contralesional corticospinal tracts following perinatal brain injury. Dev Med Child Neurol 2013;55:707-12.

26. Leemans A, Jeurissen B, Sijbers J, and Jones DK. ExploreDTI: a graphical toolbox for processing, analyzing, and visualizing diffusion MR data. In: 17th Annual Meeting of Intl Soc Mag Reson Med, p. 3537, Hawaii, USA, 2009.

27. Leemans A, Jones DK. The B-matrix must be rotated when correcting for subject motion in DTI data. Magn Reson Med 2009;61:1336-49.

28. Tax CM, Otte WM, Viergever MA, Dijkhuizen RM, Leemans A. REKINDLE: robust extraction of kurtosis INDices with linear estimation. Magn Reson Med 2015;73:794-808.

29. Veraart J, Sijbers J, Sunaert S, Leemans A, Jeurissen B. Weighted linear least squares estimation of diffusion MRI parameters: strengths, limitations, and pitfalls. Neuroimage 2013;81:335-46.

30. Oishi K, Mori S, Donohue PK, et al. Multi-contrast human neonatal brain atlas: application to normal neonate development analysis. Neuroimage 2011;56:8-20.

31. Klein S, Staring M, Murphy K, Viergever MA, Pluim JP. elastix: a toolbox for intensity-based medical image registration. IEEE Trans Med Imaging 2010;29:196-205.

32. Rosenbaum P, Paneth N, Leviton A, et al. A report: the definition and classification of cerebral palsy April 2006. Dev Med Child Neurol Suppl 2007;109:8-14.

33. Reich DS, Smith SA, Jones CK, et al. Quantitative characterization of the corticospinal tract at 3T. AJNR Am J Neuroradiol 2006;27:2168-78.

34. Youden WJ. Index for rating diagnostic tests. Cancer 1950;3:32-5. 